\title{
Association between FOXM1 and hedgehog signaling pathway in human cervical carcinoma by tissue microarray analysis
}

\author{
HONG CHEN, JINGJING WANG, HONG YANG, DAN CHEN and PANPAN LI
}

Department of Gynecology and Obstetrics, Zhongnan Hospital of Wuhan University, Wuhan, Hubei 430071, P.R. China

Received January 28, 2015; Accepted February 19, 2016

DOI: $10.3892 / \mathrm{ol} .2016 .4932$

\begin{abstract}
Forkhead box M1 (FOXM1) and hedgehog (Hh) signaling pathway are implicated in the formation and development of human tumors, including cervical cancer. Previous studies have indicated that FOXM1 may be a downstream target gene of the Hh signaling pathway, but their association in cervical cancer is largely unknown. In the present study, the expression of FOXM1 and Hh signaling molecules was evaluated by immunohistochemical analysis in a tissue microarray that contained 70 cervical cancer tissues and 10 normal cervical tissues. In addition, the association of these molecules with clinicopathological parameters, and the association between FOXM1 and various molecules involved in the Hh signaling pathway was investigated. The results indicated that FOXM1 and Hh signaling molecules were overexpressed in cervical cancer tissues. The protein expression levels of FOXM1, glioma-associated oncogene 1 (GLI1) and smoothened (SMO) correlated with the clinical stage of the tumors, while the protein expression levels of Sonic Hh (SHh), patched 1 (PTCH1) and GLI1 correlated with the pathological grade of the tumors. The expression levels of GLI1 were lower in tissues without lymph node metastasis than in tissues with lymph node metastasis. In addition, FOXM1 expression correlated with GLI1, SHh and PTCH1 expression in cancer tissues. These findings confirmed the participation of FOXM1 and the Hh signaling pathway in cervical cancer. Furthermore, the finding that FOXM1 may be a downstream target gene of the Hh signaling pathway in cervical cancer provides a potential novel diagnostic and therapeutic target for cervical cancer.
\end{abstract}

\section{Introduction}

Despite the improvements in diagnostic and screening techniques in recent years, and the increased availability in

Correspondence to: Dr Hong Chen, Department of Gynecology and Obstetrics, Zhongnan Hospital of Wuhan University, 169 Dong Hu Street, Wuhan, Hubei 430071, P.R. China

E-mail: znchgg@163.com

Key words: cervical cancer, FOXM1, invasion, metastasis, hedgehog signaling pathway vaccines, cervical cancer remains the second most common type of cancer affecting the female reproductive system (1), and the fourth leading cause of cancer-associated mortalities in women worldwide (2). Epidemiological studies have indicated that human papillomavirus is an important risk factor, and possibly the most required etiological agent, in the development of cervical cancer (3-5). However, this agent alone is insufficient to trigger cervical cancer development (6). Thus, a number of previous studies have speculated that other genetic events may affect this malignant transformation (7-10). However, the genetic basis underlying cervical tumorigenesis and progression is largely unknown.

The mammalian transcription factor forkhead box M1 (FOXM1) belongs to the extensive family of forkhead transcription factors, which harbor 100 amino acids and an evolutionarily conserved DNA binding domain called forkhead or winged-helix domain (11-13). FOXM1 is a dynamic cancer-associated biomarker involved in the regulation of various biological processes, including cell cycle progression, differentiation, metastasis, invasion and angiogenesis (14-18). In a previous study, the present authors demonstrated that FOXM1 was overexpressed in cervical cancer tissues, and its nuclear expression was observed to be correlated with the pathological grade of the tumor (19). Furthermore, FOXM1 may be involved in the regulation of cancer invasion and metastasis by regulating the expression and activity of matrix metalloproteinase (MMP)-2, MMP-9 and vascular endothelial growth factor (VEGF) (17). Chan et al (20) and He et al (21) also indicated that FOXM1 is important in the tumorigenesis and development of cervical cancer. However, the molecular mechanism underlying the modulation of FOXM1 expression remains unclear. Teh et al (22) demonstrated that the overexpression of glioma-associated oncogene 1 (GLI1) significantly elevated the messenger RNA (mRNA) levels and transcriptional activity of FOXM1 in numerous human tumor cell lines. Since then, various studies have attempted to identify an association between FOXM1 and hedgehog ( $\mathrm{Hh})$ signaling in human tumors (23-25).

The Hh signaling pathway, which was first reported by Nüsslein-Volhard and Wieschaus in 1980 (26), regulates growth and patterning during organogenesis, and its malfunction leads to multiple human disorders, including birth defects and cancer (27-29). In humans, this signaling pathway involves three ligands, namely Sonic Hh (SHh), Indian Hh (IHh) and Desert Hh (DHh). Among these three ligands, $\mathrm{SHh}$ is the most 
widely expressed in mammalian tissues, while IHh is specific for bone development and DHh expression is restricted to gonads $(29,30)$. The receptor for Hh ligands is a protein with 12 transmembrane domains termed patched 1 (PTCH1), which is located in the cell membrane (31). Another transmembrane protein called smoothened (SMO) is also involved in the $\mathrm{Hh}$ signaling pathway, while GLIs are zinc-finger proteins that function as translational modulators of this pathway (32).

In the absence of Hh ligands, $\mathrm{PTCH} 1$ inhibits the activity of SMO, while the binding of Hh ligands to PTCH1 relieves the inhibition of SMO caused by PTCH1, and SMO subsequently activates the translational modulators GLI1, GLI2 and GLI3 (33). This cascade of events ultimately results in the regulation of the corresponding target genes (33). Certain components of the Hh signaling pathway such as PTCH1 and GLI1 are direct transcriptional targets, thus establishing a feedback loop that regulates the level of activity of this pathway (34).

Various studies on the role of the Hh signaling pathway in cervical cancer have been previously conducted, but the precise molecular mechanism underlying the processes of metastasis and invasion in cervical cancer remains unclear. In addition, the association between the Hh signaling pathway and FOXM1 in cervical cancer is largely unknown. Therefore, the expression of $\mathrm{Hh}$ signaling molecules (including $\mathrm{SHh}$, PTCH1, SMO and GLI1) and FOXM1 was analyzed in the present study in a tissue microarray of cervical cancer using immunochemistry. In addition, the association between these molecules and the clinicopathological parameters of patients with cervical cancer (including pathological grade, clinical stage and lymph node metastasis), as well as the association between the Hh pathway and FOXM1 expression were also evaluated in the present study.

\section{Materials and methods}

Patients and tissue microarray. All patients underwent surgery in Tongxiang People's Hospital, Tongxiang, China, between January 2002 and December 2012. The patients with cervical cancer underwent a hysterectomy, while all the normal cervical samples were obtained by cervical local excision. The study was approved by the ethics committee of Tongxiang People's Hospital. Tissue microarray analysis of 80 specimens obtained from patients who underwent surgery was performed by Alenabio, Inc. (Xi'an, China). All specimens were fixed with formalin (Guangzhou Wexis Biotech Ltd., Guangzhou, China) postoperatively, and then embedded with paraffin (Jinan Shenghe Chemical Co., Ltd., Jinan, China) prior to being converted into tissue microarray slides (core size, $1.50 \mathrm{~mm}$; 10x8 rows). The slides were analyzed for histological type and tumor grade by two pathologists (Dr Fang Yu and Dr Sufang Tian, Zhongnan Hospital of Wuhan University, Wuhan, China). Histopathological examination revealed that the specimens consisted of 4 adenocarcinomas, 66 squamous cell carcinomas and 10 normal cases. Of the 70 tumors, $4(5.71 \%)$ were grade I, $43(61.43 \%)$ were grade II, $19(27.14 \%)$ were grade III, and 4 (5.71\%) were undetermined. The clinical stage classification of the tumors was based on the 7 th edition of the cancer staging manual published by the American Joint Committee on Cancer (AJCC), and revealed that of the
70 tumors, $22(31.43 \%)$ were stage I, 20 (28.57\%) were stage II and $28(40.00 \%)$ were stage III. The lymph node status was determined according to the tumor-node-metastasis classification criterion, and indicated that lymph node metastasis was present in $44(62.86 \%)$ cases. All the information regarding the tumor specimens is listed in Table I. Written consent was obtained from all patients.

Immunohistochemistry. Tissue sections were deparaffinized with serially decreasing concentrations of ethanol (Sinopharm Group Co., Ltd., Shanghai China) and then rehydrated in distilled water for $2 \mathrm{~min}$. For antigen retrieval, the slides were pretreated with $0.01 \mathrm{M}$ citrate buffer $\mathrm{pH} 6.0$ (Thermo Fisher Scientific, Inc., Waltham, MA, USA), prior to be heated in a KJ23B microwave oven (Midea, Shunde, China) for $15 \mathrm{~min}$. To detect SMO, GLI1 and FOXM1 antigens, the endogenous peroxidase activity was blocked with $3 \% \mathrm{H}_{2} \mathrm{O}_{2}$ (Nanjing Senbeijia Biological Technology Co., Ltd., Nanjing, China) in methanol (Shanghai Macklin Biochemical Co., Ltd., Shanghai, China) for $15 \mathrm{~min}$ at room temperature, and the sections were then incubated with the corresponding primary antibodies overnight at $4^{\circ} \mathrm{C}$. Subsequently, the sections were incubated with peroxidase-conjugated secondary antibodies (catalogue no. PV-9000; ZSGB Biotechnology, Beijing, China). Protein expression was visualized by the brown pigmentation resulting from the chromogen 3,3'-diaminobenzidine (DAB) hydrochloride (catalogue no. AR1022; Wuhan Boster Biological Technology, Ltd., China). Next, the slides were counterstained with hematoxylin (catalogue no. H9627, Sigma-Aldrich, St. Louis, MO, USA), rinsed several times with phosphate-buffered saline (PBS; Wuhan Boster Biological Technology, Ltd.) pH 7.4, dehydrated with a series of graded ethanol solutions, cleared using xylene (Sinopharm Group Co., Ltd.), and observed under an ECLIPSE E100 optical microscope (Nikon Corporation, Tokyo, Japan). To detect SHh and PTCH1 antigens, the endogenous peroxidase activity was blocked with rabbit serum (Shanghai Beiyi Bioequip Information Co., Ltd., Shanghai, China) for $30 \mathrm{~min}$ at room temperature, prior to incubating the tissue sections with the corresponding primary antibodies overnight at $4^{\circ} \mathrm{C}$. The primary antibodies used were as follows: Goat anti-SHh polyclonal antibody (catalogue no. sc-1194; 1:120 dilution), goat anti-PTCH1 polyclonal antibody (catalogue no. sc-6149; 1:120 dilution), rabbit anti-SMO polyclonal antibody (catalogue no. sc-13943; 1:120 dilution), rabbit anti-GLI1 polyclonal antibody (catalogue no. sc-20687; 1:100 dilution) (Santa Cruz Biotechnology, Inc., Dallas, TX, USA) and rabbit polyclonal anti-FOXM1 antibody (1:150 dilution; ProteinTech Group, Inc., Chicago, IL, USA). Next, biotinylated rabbit anti-goat immunoglobulin G (catalogue no. SA1023; Wuhan Boster Biological Technology) was added to the sections and incubated at $30^{\circ} \mathrm{C}$ for $30 \mathrm{~min}$. The immunoreactivity of the tissue sections was visualized using DAB hydrochloride. Sections were then counterstained with hematoxylin, rinsed in PBS pH 7.4, dehydrated with ethanol, cleared with xylene, and observed under an ECLIPSE E100 optical microscope.

Quantitative analysis. The slides were examined under an ECLIPSE E100 microscope. Positive cells were stained as brownish granules in the cytoplasm or in the nucleus. The staining intensity for the Hh signaling molecules and 
Table I. Clinicopathological characteristics of 70 tumor cases.

\begin{tabular}{llc}
\hline Characteristics & Adenocarcinoma, $(\%)$ & Squamous cell carcinoma, $\mathrm{n}(\%)$ \\
\hline Total & $4(5.71)$ & $66(94.29)$ \\
Pathological stage & & $4(6.06)$ \\
Well differentiated & $0(0.00)$ & $40(60.61)$ \\
Moderately differentiated & $3(75.00)$ & $19(28.79)$ \\
Poorly differentiated & $0(0.00)$ & $3(4.54)$ \\
Unclear & $1(25.00)$ & $31(46.97)$ \\
Invasive extent & & $35(53.03)$ \\
Confined to uterus & $1(25.00)$ & \\
Beyond the uterus & $3(75.00)$ & $41(62.12)$ \\
Lymph node status & & $24(36.36)$ \\
N0 & $3(75.00)$ & $1(1.52)$ \\
N1 & $0(0.00)$ & $18(27.27)$ \\
Unclear & $1(25.00)$ & $40(60.61)$ \\
Age, years & & $8(12.12)$ \\
$\leq 40$ & $2(50.00)$ & $1(25.00)$ \\
$41-56$ & $1(25.00)$ & \\
$\geq 56$ & & \\
\hline
\end{tabular}

Table II. Comparison of FOXM1 and Hh signaling molecules in tumors and normal tissues.

\begin{tabular}{|c|c|c|c|c|c|}
\hline \multirow[b]{2}{*}{ Protein } & \multicolumn{2}{|c|}{ Squamous cell carcinoma } & \multicolumn{2}{|c|}{ Adenocarcinoma } & \multirow[b]{2}{*}{ P-value ${ }^{c}$} \\
\hline & $\mathrm{IOD}($ mean $\pm \mathrm{SD})$ & P-value ${ }^{a}$ & $\mathrm{IOD}($ mean $\pm \mathrm{SD})$ & P-value ${ }^{b}$ & \\
\hline FOXM1 & $(2.27 \pm 1.64) \times 10^{5}$ & $<0.001$ & $(1.39 \pm 1.17) \times 10^{5}$ & 0.014 & 0.340 \\
\hline $\mathrm{SHh}$ & $(3.27 \pm 0.95) \times 10^{5}$ & $<0.001$ & $(3.38 \pm 0.37) \times 10^{5}$ & 0.007 & 0.743 \\
\hline SMO & $(1.89 \pm 1.16) \times 10^{5}$ & $<0.001$ & $(1.58 \pm 0.84) \times 10^{5}$ & 0.007 & 0.743 \\
\hline GLI1 & $(1.33 \pm 0.96) \times 10^{5}$ & $<0.001$ & $(1.41 \pm 0.73) \times 10^{5}$ & 0.014 & 0.620 \\
\hline PTCH1 & $(1.71 \pm 0.58) \times 10^{5}$ & $<0.001$ & $(1.97 \pm 0.17) \times 10^{5}$ & 0.007 & 0.236 \\
\hline
\end{tabular}

${ }^{a}$ Squamous cell carcinoma compared with normal tissues. ${ }^{b}$ Adenocarcinoma compared with normal tissues. ${ }^{c}$ Squamous cell carcinoma compared with adenocarcinoma. FOXM1, forkhead box M1; Hh, hedgehog; SHh, Sonic Hh; SMO, smoothened; GLI1, glioma-associated oncogene 1; PTCH1, patched 1; IOD, integrated optical density; SD, standard deviation.

FOXM1 protein in cervical tumors and normal epithelia was semi-quantitatively assessed. The immunostaining densities of each point of the tissue microarray were quantitatively assessed with Image-Pro Plus version 6.0 (Media Cybernetics, Inc., Rockville, MD, USA). Briefly, the sections were placed under the microscope and photographed. The images were then transferred via a digital camera (EOS 600D; Canon, Inc., Tokyo, Japan) to a computer. A total of three separate positive areas at $\times 400$ magnification were selected in each point of the immunostaining sections, and the integrated optical density (IOD) was measured. The IOD values obtained in three areas were averaged and used to calculate the mean values.

Statistical analysis. Statistical analyses were performed with SPSS 20.0 software (IBM SPSS, Armonk, NY, USA). The association between the protein expression levels (according to the IOD values) of FOXM1 and Hh signaling molecules and the clinicopathological parameters of the tumors was evaluated with independent $t$-tests in order to compare the differences between two groups. To compare differences between $\geq 2$ groups, one-way analysis of variance was used for those cases of equal variance, while Kruskal-Wallis test was used for unequal variance. The correlation between the expression levels of each protein was analyzed with the Spearman's rank-order correlation test. Data were reported as the mean \pm standard deviation. $\mathrm{P}<0.05$ was considered to indicate a statistically significant difference.

\section{Results}

Overall data. The 80 specimens analyzed in the present study consisted of 70 cancer cases and 10 normal cases. The mean age of the patients was 44 years (range, 15-72 years). The expression levels of the aforementioned proteins were 
Table III. Association between FOXM1 expression and clinicopathological characteristics.

\begin{tabular}{|c|c|c|c|}
\hline Characteristics & Patients, n (\%) & $\mathrm{IOD}($ mean $\pm \mathrm{SD})$ & P-value \\
\hline \multicolumn{4}{|l|}{ Pathological stage $^{a}$} \\
\hline Well/moderately differentiated & $47(67.14)$ & $(2.18 \pm 1.72) \times 10^{5}$ & 0.512 \\
\hline Poorly differentiated & $19(27.14)$ & $(2.48 \pm 1.45) \times 10^{5}$ & \\
\hline \multicolumn{4}{|l|}{ Invasive extent ${ }^{\mathrm{b}}$} \\
\hline Stage 1 & $22(31.43)$ & $(1.54 \pm 1.49) \times 10^{5}$ & $0.011^{\mathrm{c}}$ \\
\hline Stage 2 & $20(28.57)$ & $(2.34 \pm 1.36) \times 10^{5}$ & $0.103^{\mathrm{d}}$ \\
\hline Stage 3 & $28(40.00)$ & $(2.71 \pm 1.75) \times 10^{5}$ & $0.421^{\mathrm{e}}$ \\
\hline \multicolumn{4}{|l|}{ Lymph node status ${ }^{\mathrm{f}}$} \\
\hline N0 & $44(62.86)$ & $(2.09 \pm 1.60) \times 10^{5}$ & 0.408 \\
\hline N1 & $24(34.29)$ & $(2.44 \pm 1.72) \times 10^{5}$ & \\
\hline \multicolumn{4}{|l|}{ Age, years } \\
\hline$\leq 40$ & $20(28.57)$ & $(2.72 \pm 1.71) \times 10^{5}$ & $0.033^{\mathrm{g}}$ \\
\hline $41-56$ & $41(58.57)$ & $(2.20 \pm 1.63) \times 10^{5}$ & $0.230^{\mathrm{h}}$ \\
\hline$\geq 56$ & $9(12.86)$ & $(1.33 \pm 0.99) \times 10^{5}$ & $0.143^{\mathrm{i}}$ \\
\hline
\end{tabular}

${ }^{a}$ Pathological stage was undetermined in 4 patients. ${ }^{b}$ According to the American Joint Committee on Cancer classification. Equal variance,

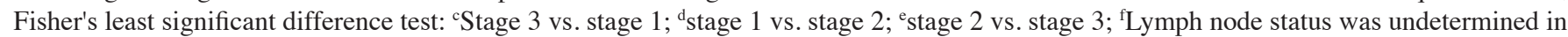

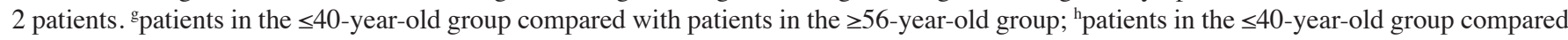
with patients in the 41-56 years old group; ipatients in the 41-56-year-old compared with patients in the $\geq 56$-year-old group. IOD, integrated optical density; SD, standard deviation.

Table IV. Association between SHh and PTCH1 expression and clinicopathological characteristics.

\begin{tabular}{|c|c|c|c|c|c|}
\hline \multirow[b]{2}{*}{ Characteristics } & \multirow[b]{2}{*}{ Patients, n (\%) } & \multicolumn{2}{|l|}{ SHh } & \multicolumn{2}{|c|}{ PTCH1 } \\
\hline & & $\mathrm{IOD}($ mean $\pm \mathrm{SD})$ & P-value & $\mathrm{IOD}($ mean $\pm \mathrm{SD})$ & P-value \\
\hline \multicolumn{6}{|l|}{ Pathological grade ${ }^{a}$} \\
\hline Well/moderately differentiated & $47(67.14)$ & $(2.98 \pm 0.82) \times 10^{5}$ & $<0.001^{\mathrm{a}}$ & $(1.60 \pm 0.49) \times 10^{5}$ & $0.023^{\mathrm{b}}$ \\
\hline Poorly differentiated & $19(27.14)$ & $(3.95 \pm 0.92) \times 10^{5}$ & & $(1.96 \pm 0.73) \times 10^{5}$ & \\
\hline \multicolumn{6}{|l|}{ Invasive extent ${ }^{c}$} \\
\hline Stage 1 & $22(31.43)$ & $(3.04 \pm 0.83) \times 10^{5}$ & $0.463^{\mathrm{d}}$ & $(1.81 \pm 0.57) \times 10^{5}$ & $0.473^{\mathrm{d}}$ \\
\hline Stage 2 & $20(28.57)$ & $(3.25 \pm 1.06) \times 10^{5}$ & $0.413^{\mathrm{e}}$ & $(1.68 \pm 0.38) \times 10^{5}$ & $0.911^{\mathrm{e}}$ \\
\hline Stage 3 & $28(40.00)$ & $(3.47 \pm 0.94) \times 10^{5}$ & $0.104^{\mathrm{f}}$ & $(1.66 \pm 0.69) \times 10^{5}$ & $0.373^{\mathrm{f}}$ \\
\hline \multicolumn{6}{|l|}{ Lymph node status ${ }^{\mathrm{g}}$} \\
\hline No & $44(62.86)$ & $(3.13 \pm 0.87) \times 10^{5}$ & 0.113 & $(1.75 \pm 0.50) \times 10^{5}$ & 0.528 \\
\hline N1 & $24(34.29)$ & $(3.52 \pm 1.05) \times 10^{5}$ & & $(1.66 \pm 0.72) \times 10^{5}$ & \\
\hline \multicolumn{6}{|l|}{ Age, years } \\
\hline$\leq 40$ & $20(28.57)$ & $(3.19 \pm 1.06) \times 10^{5}$ & $0.601^{\mathrm{h}}$ & $(1.74 \pm 0.54) \times 10^{5}$ & $0.802^{\mathrm{k}}$ \\
\hline $41-56$ & $41(58.57)$ & $(3.32 \pm 0.96) \times 10^{5}$ & $0.767^{\mathrm{i}}$ & $(1.67 \pm 0.44) \times 10^{5}$ & \\
\hline$\geq 56$ & $9(12.86)$ & $(3.27 \pm 0.95) \times 10^{5}$ & $0.933^{\mathrm{j}}$ & $(1.86 \pm 0.11) \times 10^{5}$ & \\
\hline
\end{tabular}

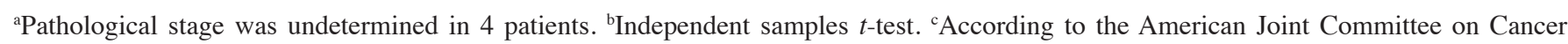
classification. Equal variance, Fisher's least significant difference test: ${ }^{\mathrm{d}}$ Stage 1 vs. stage 2; ${ }^{\mathrm{e}}$ stage 2 vs. stage 3; ${ }^{\mathrm{f}}$ stage 1 vs. stage 3; ${ }^{\mathrm{g}} \mathrm{Lymph}$ node status was undetermined in 2 patients. ${ }^{\text {h}}$ patients in the $\leq 40$-year-old group compared with patients in the 41-56-year-old group; i patients in the 41-56-year-old compared with patients in the $\geq 56$-year-old group; ${ }^{j}$ patients in the $\leq 40$-year-old group compared with patients in the 256-year-old group. ${ }^{k}$ Unequal variance, Kruskal-Wallis test. SHh, Sonic hedgehog; PTCH1, patched 1; IOD, integrated optical density; SD, standard deviation.

significantly higher in the tumor specimens than in normal tissues. No significant differences were detected between the squamous cell carcinoma tissues and adenocarcinoma tissues (Table II). Tumor cases were divided into three groups, based 
Table V. Association between GLI1 and SMO expression and clinicopathological characteristics.

\begin{tabular}{|c|c|c|c|c|c|}
\hline \multirow[b]{2}{*}{ Characteristics } & \multirow[b]{2}{*}{ Patients, n (\%) } & \multicolumn{2}{|l|}{ GLI1 } & \multicolumn{2}{|c|}{ SMO } \\
\hline & & IOD $($ mean \pm SD $)$ & P-value & $\mathrm{IOD}($ mean $\pm \mathrm{SD})$ & P-value \\
\hline \multicolumn{6}{|l|}{ Pathological stage $\mathrm{e}^{\mathrm{a}}$} \\
\hline Well/moderately differentiated & $47(67.14)$ & $(1.18 \pm 0.94) \times 10^{5}$ & $0.022^{\mathrm{b}}$ & $(1.78 \pm 1.05) \times 10^{5}$ & 0.322 \\
\hline Poorly differentiated & $19(27.14)$ & $(1.77 \pm 0.90) \times 10^{5}$ & & $(2.10 \pm 1.36) \times 10^{5}$ & \\
\hline \multicolumn{6}{|l|}{ Invasive extent $^{c}$} \\
\hline Stage 1 & $22(31.43)$ & $(8.89 \pm 6.87) \times 10^{4}$ & $0.005^{\mathrm{d}}$ & $(2.40 \pm 1.51) \times 10^{5}$ & $0.019^{\mathrm{d}}$ \\
\hline Stage 2 & $20(28.57)$ & $(1.33 \pm 0.53) \times 10^{5}$ & & $(1.26 \pm 0.55) \times 10^{5}$ & \\
\hline Stage 3 & $28(40.00)$ & $(1.69 \pm 1.20) \times 10^{5}$ & & $(1.91 \pm 0.92) \times 10^{5}$ & \\
\hline \multicolumn{6}{|l|}{ Lymph node status ${ }^{\mathrm{e}}$} \\
\hline N0 & $44(62.86)$ & $(1.15 \pm 0.73) \times 10^{5}$ & $0.017^{\mathrm{b}}$ & $(1.92 \pm 1.26) \times 10^{5}$ & 0.853 \\
\hline N1 & $24(34.29)$ & $(1.72 \pm 1.20) \times 10^{5}$ & & $(1.87 \pm 0.95) \times 10^{5}$ & \\
\hline \multicolumn{6}{|l|}{ Age, years } \\
\hline$\leq 40$ & $20(28.57)$ & $(1.25 \pm 0.89) \times 10^{5}$ & $0.981^{f}$ & $(1.90 \pm 1.33) \times 10^{5}$ & $0.747^{\mathrm{f}}$ \\
\hline $41-56$ & $41(58.57)$ & $(1.36 \pm 1.01) \times 10^{5}$ & $0.783^{\mathrm{g}}$ & $(1.80 \pm 1.11) \times 10^{5}$ & $0.567^{\mathrm{g}}$ \\
\hline$\geq 56$ & $9(12.86)$ & $(1.42 \pm 0.84) \times 10^{5}$ & $0.788^{\mathrm{h}}$ & $(2.17 \pm 0.90) \times 10^{5}$ & $0.389^{\mathrm{h}}$ \\
\hline
\end{tabular}
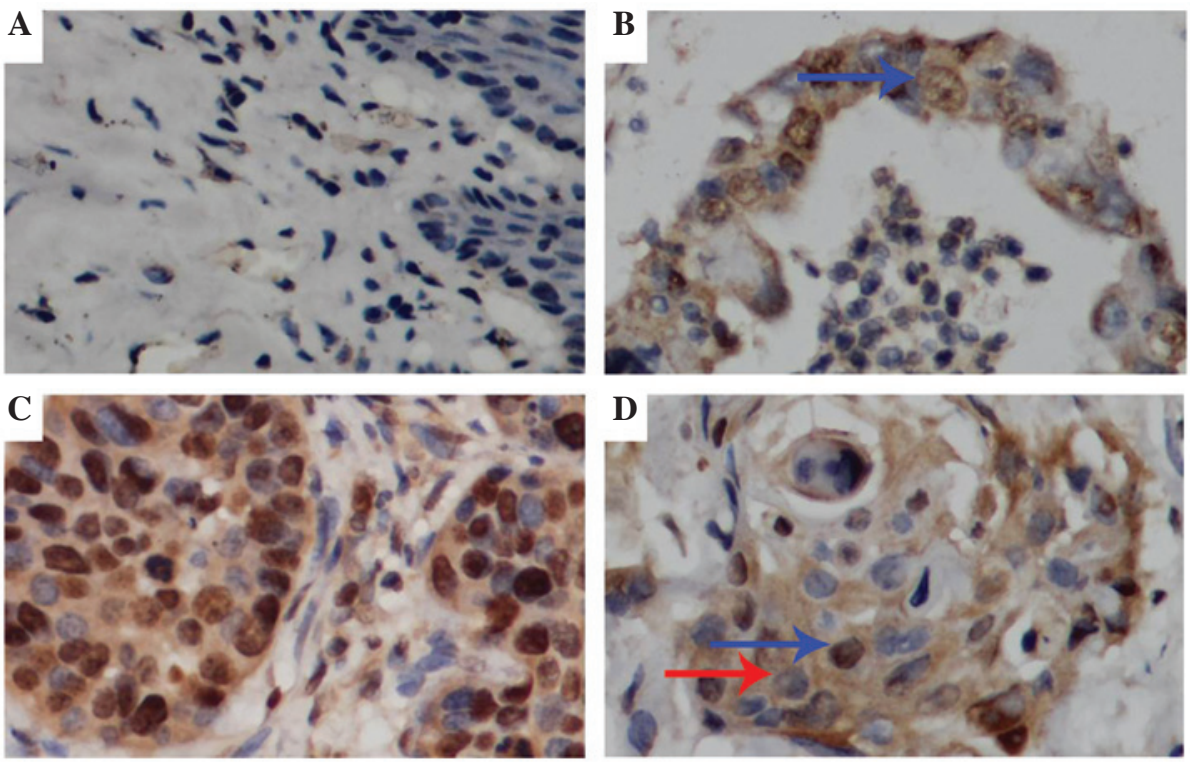

Figure 1. (A) Negative staining for FOXM1 expression in normal cervical tissue, compared with overexpression of FOXM1 in cervical cancer. FOXM1 was overexpressed in (B) adenocarcinoma and (C and D) squamous cell carcinoma (2 examples). FOXM1 expression was detected both in the cytoplasm (red arrow) and nucleus (blue arrow) of cervical cancer cells. Magnification, x400. FOXM1, forkhead box M1.

on patients' age ( $\leq 40,41-56$ and $\geq 56$ years, respectively). No significant correlation was observed between protein expression and patient age, except for FOXM1 expression in $<41$ and $>55$-year-old patients $(\mathrm{P}=0.033)$. No significant correlation was observed between the expression of FOXM1, SHh, PTCH1 or SMO and lymph node status $(\mathrm{P}=408$, $\mathrm{P}=0.113, \mathrm{P}=0.528$ and $\mathrm{P}=0.853$, respectively).
FOXM1 expression in cervical cancer and normal tissues. FOXM1 protein was stained as brownish granules in the cytoplasm and particularly in the nucleus of positive cells (Fig. 1). In the control samples, the background was clear with no specific staining. Table III shows the expression of FOXM1 in cervical cancer tissues. The IOD value of FOXM1 in normal cases was $(2.97 \pm 1.67) \times 10^{4}$. According to the AJCC staging classification 
$\mathrm{SHh}$
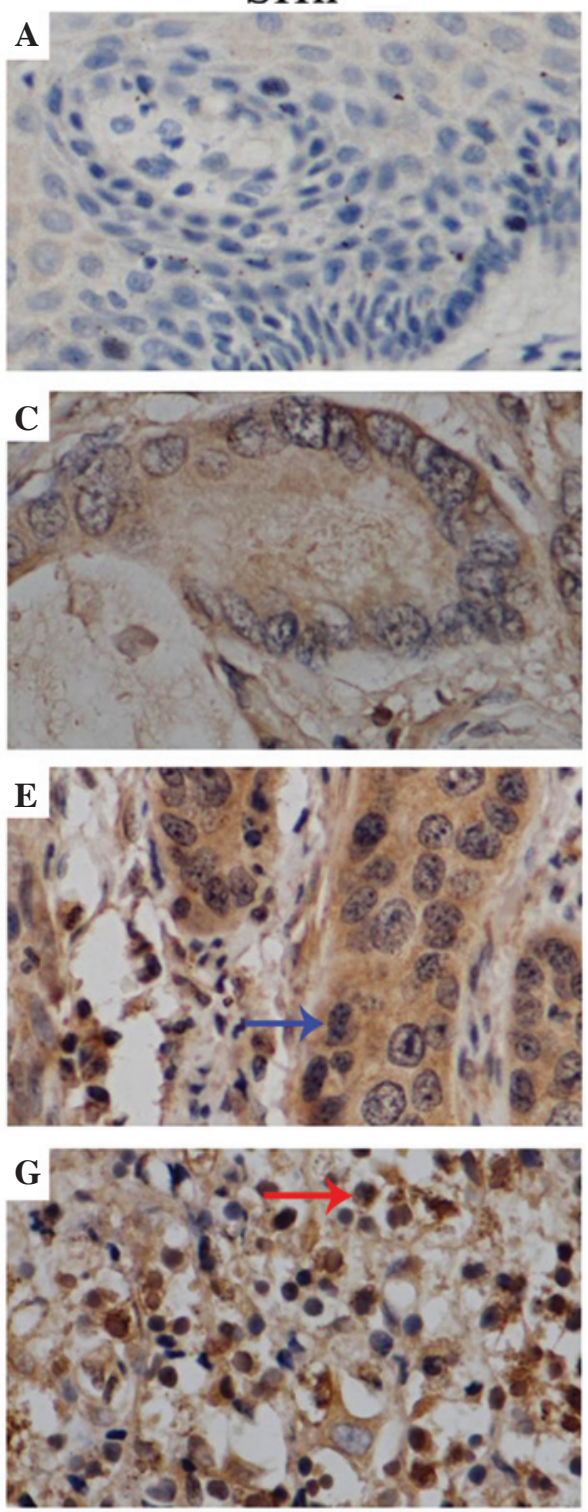

PTCH1
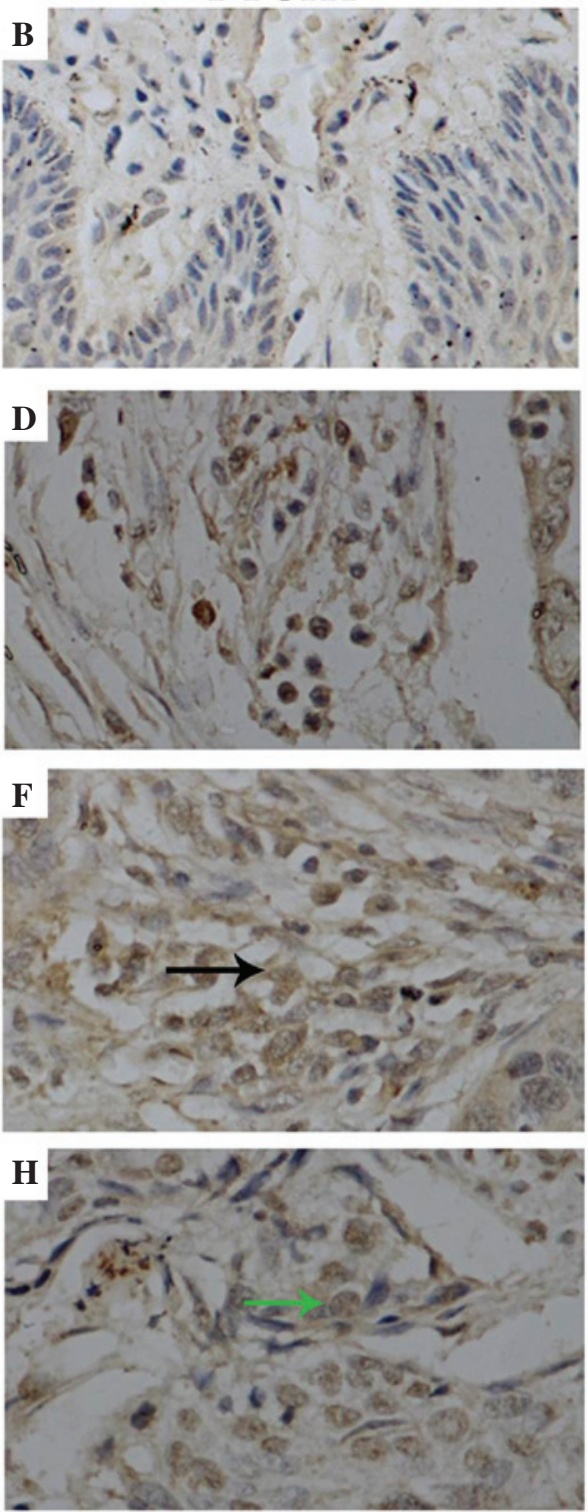

Figure 2. Overexpression of SHh and PTCH1 in cervical cancer. (A) Negative immunostaining of SHh in normal cervical tissue. (B) Negative immunostaining of PTCH1 in normal cervical tissue. (C) Increased expression of SHh was observed in adenocarcinoma. (D) Increased expression of PTCH1 was observed in adenocarcinoma. (E and G) Increased expression of SHh was observed in squamous cell carcinoma (2 examples). SHh expression was detected in the (E) cytoplasm (blue arrow) and (G) nucleus (red arrow) of cervical cancer cells. (F and H) Increased expression of PTCH1 was observed in squamous cell carcinoma (2 examples). PTCH1 expression was detected in the (F) cytoplasm (black arrow) and (H) nucleus (green arrow) of cervical cancer cells. Magnification, $\mathrm{x} 400$. SHh, Sonic hedgehog; PTCH1, patched 1.

system, FOXM1 protein expression differed at different tumor stages $(\mathrm{P}=0.011)$, but did not exhibit any significant correlation with pathological grade. In addition, FOXM1 expression exhibited no correlation with the lymph node metastasis status of cervical cancer. Spearman's rank-order correlation test indicated that FOXM1 expression correlated with GLI1 $(\mathrm{R}=0.405$, $\mathrm{P}<0.001)$, SHh $(\mathrm{R}=0.416, \mathrm{P}<0.001)$ and PTCH1 $(\mathrm{R}=0.281$, $\mathrm{P}=0.012$ ) expression.

Expression levels of SHh and PTCH1 in cervical cancer and normal tissues. Immunohistochemical staining identified SHh and PTCH1 in the cytoplasm and nucleus of tumor cells (Fig. 2). The IOD values of SHh and PTCH1 in normal specimens were $(8.82 \pm 3.04) \times 10^{3}$ and $(3.46 \pm 1.27) \times 10^{4}$, respectively.
The expression of SHh significantly differed between different pathological grades, and was significantly higher in poorly differentiated tumors than in moderately differentiated tumors $(\mathrm{P}<0.001)$. No difference was observed in terms of invasive extent or lymph node metastasis. The expression levels of PTCH1 were not associated with invasive extent. By contrast, the expression of PTCH1 was significantly correlated with the tumor pathological grade, being the expression levels of PTCH1 higher in poorly differentiated tissues than in moderately differentiated tissues $(\mathrm{P}=0.023)$. The results of the statistical analysis conducted for SHh and PTCH1 expression are listed in Table IV.

Expression levels of SMO and GLII in cervical cancer and normal tissues. Immunohistochemical staining identified 

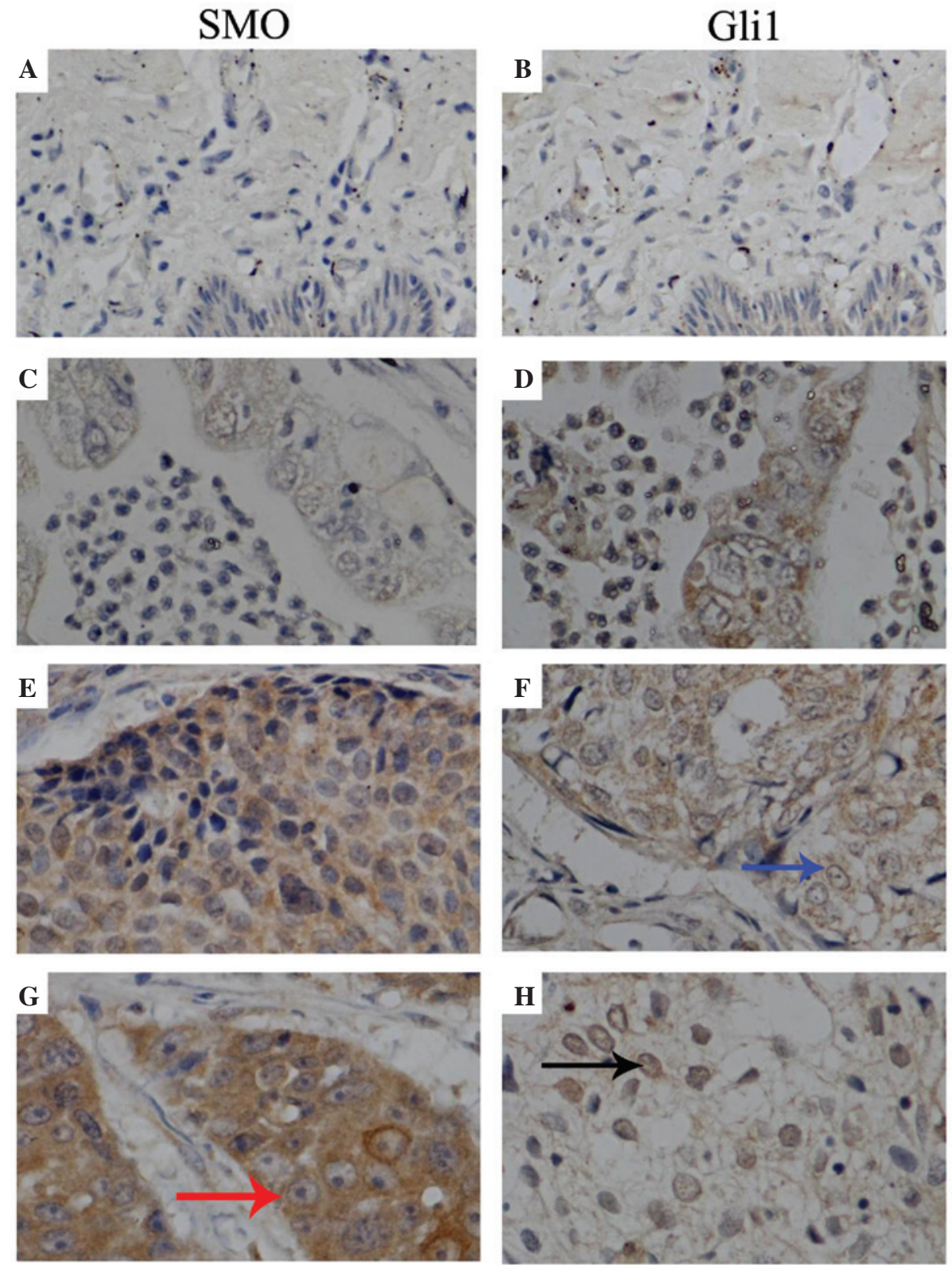

Figure 3. Overexpression of SMO and GLI1 in cervical cancer. (A) Negative staining of SMO in normal tissue. (B) Negative staining of GLI1 in normal tissue. (C) Increased expression of SMO was observed in adenocarcinoma. (D) GLI1 was highly expressed in adenocarcinoma. (E and G) Increased expression of SMO was observed in squamous cell carcinoma (2 examples). SMO was mainly localized in the cytoplasm of cervical cancer cells (red arrow). (F and H) GLI1 was highly expressed in squamous cell carcinoma (2 examples). GLI1 was expressed in the (F) cytoplasm (blue arrow) and (H) nucleus (black arrow) of cervical cancer cells. Magnification, x400. SMO, smoothened; GLI1, glioma-associated oncogene 1.

SMO mainly in the cytoplasm of tumor cells, while GLI1 was expressed both in the cytoplasm and nucleus (Fig. 3). The IOD values of SMO and GLI1 in normal specimens were $(2.41 \pm 1.43) \times 10^{4}$ and $(2.48 \pm 2.35) \times 10^{4}$, respectively. The expression of SMO was not associated with the tumor pathological grade. However, the expression levels of this protein correlated with the tumor invasive extent, as revealed by the results of Kruskal-Wallis test $(\mathrm{P}=0.019)$. The expression of GLI1 was significantly lower in moderately differentiated cervical cancer tissues than in poorly differentiated cervical cancer tissues $(\mathrm{P}=0.022)$. In addition, the expression of GLI1 correlated with the invasive extent of cervical tumors $(\mathrm{P}=0.005)$. GLI1 expression was also higher in tumors with lymph node metastasis than in tissues without lymph node metastasis $(\mathrm{P}=0.017)$. The results of the statistical analysis regarding these proteins are listed in Table $\mathrm{V}$.

\section{Discussion}

Cervical cancer is the second most common malignancy among women worldwide, and its incidence and mortality rates rank the third and fourth, respectively, among female malignant tumors (2). The metastasis capability of cervical cancer contributes to its malignance (35). Therefore, elucidation of the molecular mechanism underlying cancer formation and progression is urgently required. In the present study, the expression patterns of FOXM1 and Hh signaling molecules in cervical cancer were characterized via immunohistochemistry. 
The results indicated that FOXM1 protein and Hh signaling molecules were overexpressed in cervical cancer tissues. In addition, the association between FOXM1 and the Hh signaling pathway was also analyzed. The results indicated that FOXM1 overexpression correlated with overexpression of molecules participating in the Hh signaling pathway. These data suggest that FOXM1 is important in cervical cancer and that the $\mathrm{Hh}$ signaling pathway participates in its modulation. Overall, the present study suggests that therapies directed against FOXM1 and the Hh signaling pathway are a promising novel approach for cervical cancer treatment.

Increased expression of FOXM1 has been previously detected in diverse cancer cell lines and tissues $(36,37)$. In the present study, FOXM1 expression was significantly higher in cervical cancer tissues than in normal cervical tissues (squamous carcinoma vs. normal cervical tissue, $\mathrm{P}<0.001$; adenocarcinoma vs. normal cervical tissue, $\mathrm{P}=0.014)$. This result is in accordance with the studies by Chan et al (20) and He et al (21). The present study also revealed that the expression of FOXM1 correlated with cancer invasion $(\mathrm{P}=0.011)$, similarly to the results reported by Chan et al $(20)$ and He et al (21), who also noted an association between FOXM1 expression and tumor stage. In the current study, no association was detected between FOXM1 expression and tumor pathological grade or lymph node metastasis. However, FOXM1 expression was lower in patients older than 55 years than in patients younger than 41 years $(\mathrm{P}=0.033)$. This result suggests that FOXM1 expression decreases with age. To the best of our knowledge, the present study is the first to explore the association between FOXM1 expression and patient age. In a previous study, the present authors demonstrated that RNA interference (RNAi)-mediated FOXM1 knockdown inhibited cervical cancer migration, invasion and angiogenesis in vivo and in vitro (18). In the present study, the results of immunohistochemistry analysis indicated that FOXM1 expression also correlated with cancer invasive extent in human cervical cancer tissues. These results suggest an important function of FOXM1 in cervical cancer metastasis and invasion.

Previous studies have reported that the $\mathrm{Hh}$ signaling pathway participates in various tumor processes, including formation, development, metastasis and angiogenesis (38-40). In the present study, the expression of the molecules involved in the Hh signaling pathway was investigated, and the results revealed that GLI1, PTCH1, SMO and SHh were overexpressed in cervical cancer tissues (squamous carcinoma vs. normal cervical tissue, all $\mathrm{P}<0.01$; adenocarcinoma vs. normal cervical tissue, $\mathrm{P}=0.014, \mathrm{P}=0.007, \mathrm{P}=0.007$ and $\mathrm{P}=0.007$, respectively). In addition, the association between the expression levels of the above Hh signaling molecules and the clinicopathological characteristics of patients was also analyzed in the present study. The expression levels of SHh, PTCH1 and GLI1 were significantly higher in the poorly differentiated tumor cases than in the moderately differentiated tumor cases $(\mathrm{P}<0.001, \mathrm{P}=0.023$ and $\mathrm{P}=0.022$, respectively). The expression levels of GLI1 and SMO also correlated with the invasive extent of cervical tumors ( $\mathrm{P}=0.005$ and $\mathrm{P}=0.019$, respectively), while GLI1 expression additionally correlated with lymph node metastasis $(\mathrm{P}=0.017)$. The overexpression of these molecules, which are involved in the Hh signaling pathway, implies that this pathway participates in the formation and invasion of cervical cancer. Xuan et al (41) also studied the expression of the aforementioned Hh signaling molecules in cervical cancer, and observed that their expression was higher in carcinoma tissues and cervical intraepithelial neoplasia of stage II/III than in normal tissues. Furthermore, Samarzija and Beard (42) reported that the overexpression of GLI1, PTCH1, SMO and SHh in cervical cancer cells (including C33-A, SiHa, C4-1, CaSki and HeLa), and the inhibition of the Hh signaling pathway by its inhibitor, reduced the proliferation and survival of cervical cancer cells. Overall, the results of these studies imply that the Hh signaling pathway is important in cervical cancer.

FOXM1 is a major oncogenic transcription factor, since it mediates cancer cell cycle progression, apoptosis, angiogenesis, migration, invasion and metastasis (9,43-45). Considerable efforts have been exerted in recent years to elucidate the translation and activity of FOXM1 (46). The Hh signaling pathway may modulate the transcription of FOXM1 in human transitional cell carcinoma of the bladder (47). Teh et al (22) indicated that GLI1 overexpression in primary keratinocytes and other cell lines significantly elevated the mRNA levels and transcriptional activity of FOXM1. Thus, FOXM1 may be the target of GLI1 in basal cell carcinomas. In addition, FOXM1 overexpression in non-small cell lung carcinoma has been demonstrated to correlate with PTCH1, SMO and GLI1 expression (48). In present study, the association between FOXM1 and Hh signaling molecules was analyzed, and the results indicated that FOXM1 expression significantly correlated with GLI1 ( $R=0.405$, $\mathrm{P}<0.001)$, SHh $(\mathrm{R}=0.416, \mathrm{P}<0.001)$ and PTCH1 $(\mathrm{R}=0.281$, $\mathrm{P}=0.012$ ) expression. These data indicate that FOXM1 may be the downstream of the Hh signaling pathway in cervical cancer. To the best of our knowledge, the present study is the first to describe an association between FOXM1 and the Hh signaling pathway in cervical cancer. Considering previous and present results, it is possible to conclude that FOXM1 is a downstream target of the Hh signaling pathway in human tumors.

The mechanism responsible for the regulation of metastasis and invasion in cervical cancer is not thoroughly understood at present. MMP-2 and MMP-9 principally function in tumor invasion and migration (49), whereas VEGF is a key factor in angiogenesis (50,51). In epithelial ovarian cancer cells, FOXM1 downregulation led to reduced expression of MMP-2, MMP-9 and VEGF (52). In a previous study, the present authors used RNAi to inhibit the expression of FOXM1 in cervical cancer cells, which consequently suppressed the activity and expression of MMP-2, MMP-9 and VEGF (18). Previous studies have reported that the inhibition of the Hh signaling pathway in glioma (53) and liver cancer (54) suppressed the expression of MMP-2, MMP-9 and VEGF. FOXM1 may act as the downstream element of the Hh signaling pathway, and both FOXM1 and the Hh signaling pathway may modulate the expression and activity of MMP-2, MMP-9 and VEGF (18,54-56). Thus, it is possible to hypothesize that the Hh signaling pathway may regulate the expression and activity of MMP-2, MMP-9 and VEGF through FOXM1. Further studies are required to explore the exact mechanism underlying this regulation.

In conclusion, FOXM1 may act as the downstream target of the Hh signaling pathway. GLI1, as a translational activator of this pathway, may be responsible for the translation and activation of FOXM1. In the present study, the expression of FOXM1, GLI1 and SMO correlated with cervical cancer 
clinical stage, whereas the expression of GLI1, SHh and PTCH1 correlated with tumor pathological grade. The present results were mainly based on the analysis of cervical cancer tissues. Therefore, further experiments using cervical cancer cells and cervical cancer orthotopic implantation models should be conducted in order to understand the exact mechanism underlying the regulation of cervical cancer.

\section{References}

1. Siegel R, Ma J, Zou Z and Jemal A: Cancer Statistics, 2014. CA Cancer J Clin 64: 9-29, 2014.

2. Jemal A, Bray F, Center MM, Ferlay J, Ward E and Forman D: Global cancer statistics. CA Cancer J Clin 61: 69-90, 2011.

3. Giraldi G, Martinoli L and De Luca d'Alessandro E: The human papillomavirus vaccination: A review of the cost-effectiveness studies. Clin Ter 165: e426-e432, 2014.

4. Yang SH, Kong SK, Lee SH, Lim SY and Park CY: Human papillomavirus 18 as a poor prognostic factor in stage I-IIA cervical cancer following primary surgical treatment. Obstet Gynecol Sci 57: 492-500, 2014

5. Ludmir EB, Palta M, Zhang X, Wu Y, Willett CG and Czito BG: Incidence and prognostic impact of high-risk HPV tumor infection in cervical esophageal carcinoma. J Gastrointest Oncol 5: 401-407, 2014

6. Buitrago-Pérez A, Garaulet G, Vázquez-Carballo A, Paramio JM and García-Escudero R: Molecular signature of HPV-induced carcinogenesis: pRb, p53 and gene expression profiling. Curr Genomics 10: 26-34, 2009.

7. Saha SK and Khuda-Bukhsh AR: Berberine alters epigenetic modifications, disrupts microtubule network, and modulates HPV-18 E6-E7 oncoproteins by targeting p53 in cervical cancer cell HeLa: A mechanistic study including molecular docking. Eur J Pharmacol 744: 132-146, 2014.

8. Jaiswal N, John R, Chand V and Nag A: Oncogenic human papillomavirus 16E7 modulates SUMOylation of FOXM1b. Int J Biochem Cell Biol 58: 28-36, 2015.

9. Huang C, Qiu Z, Wang L, Peng Z, Jia Z, Logsdon CD, Le X, Wei D, Huang S and Xie K: A novel FOXM1-caveolin signaling pathway promotes pancreatic cancer invasion and metastasis. Cancer Res 72: 655-665, 2012.

10. Visnovsky J, Kudela E, Farkasova A, Balharek T, Krkoska M and Danko J: Amplification of TERT and TERC genes in cervical intraepithelial neoplasia and cervical cancer. Neuro Endocrinol Lett 35: 518-522, 2014.

11. Halasi M, Pandit B, Wang M, Nogueira V, Hay N and Gartel AL: Combination of oxidative stress and FOXM1 inhibitors induces apoptosis in cancer cells and inhibits xenograft tumor growth. Am J Pathol 183: 257-265, 2013.

12. Laoukili J, Stahl M and Medema RH: FOXM1: At the crossroads of ageing and cancer. Biochim Biophys Acta 1775: 92-102, 2007.

13. Zhao F, Siu MK, Jiang L, Tam KF, Ngan HY, Le XF, Wong OG, Wong ES, Gomes AR, Bella L, et al: Overexpression of forkhead box protein M1 (FOXM1) in ovarian cancer correlates with poor patient survival and contributes to paclitaxel resistance. PLoS One 9: e113478, 2014.

14. Khongkow P, Karunarathna U, Khongkow M, Gong C, Gomes AR, Yagüe E, Monteiro LJ, Kongsema M, Zona S, Man EP, et al: FOXM1 targets NBS1 to regulate DNA damage-induced senescence and epirubicin resistance. Oncogene 33: 4144-4155, 2014.

15. Bergamaschi A, Madak-Erdogan Z, Kim YJ, Choi YL, Lu H and Katzenellenbogen BS: The forkhead transcription factor FOXM1 promotes endocrine resistance and invasiveness in estrogen receptor-positive breast cancer by expansion of stem-like cancer cells. Breast Cancer Res 16: 436, 2014.

16. Jiang L, Wang P, Chen L and Chen H: Down-regulation of FOXM1 by thiostrepton or small interfering RNA inhibits proliferation, transformation ability and angiogenesis, and induces apoptosis of nasopharyngeal carcinoma cells. Int $\mathbf{J}$ Clin Exp Pathol 7: 5450-5460, 2014.

17. Inoguchi S, Seki N, Chiyomaru T, Ishihara T, Matsushita R, Mataki H, Itesako T, Tatarano S, Yoshino H, Goto Y, et al: Tumour-suppressive microRNA-24-1 inhibits cancer cell proliferation through targeting FOXM1 in bladder cancer. FEBS Lett 588: 3170-3179, 2014.
18. Chen H, Zou Y, Yang H, Wang J and Pan H: Downregulation of FOXM1 inhibits proliferation, invasion and angiogenesis of HeLa cells in vitro and in vivo. Int J Oncol 45: 2355-2364, 2014.

19. Guan P, Chen H, Li HJ, Duan J and Chen JY: Expression and significance of FOXM1 in human cervical cancer: A tissue micro-array study. Clin Invest Med 34: E1-E7, 2011.

20. Chan DW, Yu SY, Chiu PM, Yao KM, Liu VW, Cheung AN and Ngan HY: Over-expression of FOXM1 transcription factor is associated with cervical cancer progression and pathogenesis. J Pathol 215: 245-252, 2008.

21. He SY, Shen HW, Xu L, Zhao XH, Yuan L, Niu G, You ZS and Yao SZ: FOXM1 promotes tumor cell invasion and correlates with poor prognosis in early-stage cervical cancer. Gynecol Oncol 127: 601-610, 2012.

22. Teh MT, Wong ST, Neill GW, Ghali LR, Philpott MP and Quinn AG: FOXM1 is a downstream target of GLI1 in basal cell carcinomas. Cancer Res 62: 4773-4780, 2002.

23. Huang C, Du J and Xie K: FOXM1 and its oncogenic signaling in pancreatic cancer pathogenesis. Biochim Biophys Acta 1845: 104-116, 2014.

24. Katoh Y and Katoh M: Hedgehog target genes: Mechanisms of carcinogenesis induced by aberrant hedgehog signaling activation. Curr Mol Med 9: 873-886, 2009.

25. Shigemura K and Fujisawa M: Hedgehog signaling and urological cancers. Curr Drug Targets 16: 258-271, 2015.

26. Nüsslein-Volhard C and Wieschaus E: Mutations affecting segment number and polarity in Drosophila. Nature 287: 795-801, 1980.

27. Varjosalo M and Taipale J: Hedgehog: Functions and mechanisms. Genes Dev 22: 2454-2472, 2008.

28. Hooper JE and Scott MP: Communicating with Hedgehogs. Nat Rev Mol Cell Biol 6: 306-317, 2005.

29. Jiang $J$ and Hui CC: Hedgehog signaling in development and cancer. Dev Cell 15: 801-812, 2008.

30. Laurendeau I, Ferrer M, Garrido D, D'Haene N, Ciavarelli P, Basso A, Vidaud M, Bieche I, Salmon I and Szijan I: Gene expression profiling of the hedgehog signaling pathway in human meningiomas. Mol Med 16: 262-270, 2010.

31. Rimkus TK, Carpenter RL, Qasem S, Chan M and Lo HW: Targeting the sonic hedgehog signaling pathway: Review of smoothened and GLI inhibitors. Cancers (Basel) 8: E22, 2016.

32. Callahan BP and Wang C: Hedgehog cholesterolysis: Specialized gatekeeper to oncogenic signaling. Cancers (Basel) 7: 2037-2053, 2015.

33. Rovida E and Stecca B: Mitogen-activated protein kinases and Hedgehog-GLI signaling in cancer: A crosstalk providing therapeutic opportunities? Semin Cancer Biol 35: 154-167, 2015.

34. Mathew E, Zhang Y, Holtz AM, Kane KT, Song JY, Allen BL and Pasca di Magliano M: Dosage-dependent regulation of pancreatic cancer growth and angiogenesis by hedgehog signaling. Cell Reports 9: 484-494, 2014.

35. Fan D, Wang Y, Qi P, Chen Y, Xu P, Yang X, Jin X and Tian X: MicroRNA-183 functions as the tumor suppressor via inhibiting cellular invasion and metastasis by targeting MMP-9 in cervical cancer. Gynecol Oncol: S0090-8258(16)30032, 2016 (Epub ahead of print).

36. Halasi M and Gartel AL: FOX(M1) news - it is cancer. Mol Cancer Ther 12: 245-254, 2013.

37. Kalin TV, Ustiyan V and Kalinichenko VV: Multiple faces of FOXM1 transcription factor: Lessons from transgenic mouse models. Cell Cycle 10: 396-405, 2011.

38. Shin K, Lim A, Zhao C, Sahoo D, Pan Y, Spiekerkoetter E, Liao JC and Beachy PA: Hedgehog signaling restrains bladder cancer progression by eliciting stromal production of urothelial differentiation factors. Cancer Cell 26: 521-533, 2014.

39. Sabol M, Trnski D, Uzarevic Z, Ozretic P, Musani V, Rafaj M, Cindric $M$ and Levanat S: Combination of cyclopamine and tamoxifen promotes survival and migration of mcf-7 breast cancer cells - interaction of hedgehog-gli and estrogen receptor signaling pathways. PLoS One 9: e114510, 2014.

40. Kai K, Aishima S and Miyazaki K: Gallbladder cancer: Clinical and pathological approach. World J Clin Cases 2: 515-521, 2014.

41. Xuan YH, Jung HS, Choi YL, Shin YK, Kim HJ, Kim KH, Kim WJ, Lee YJ and Kim SH: Enhanced expression of hedgehog signaling molecules in squamous cell carcinoma of uterine cervix and its precursor lesions. Mod Pathol 19: 1139-1147, 2006.

42. Samarzija I and Beard P: Hedgehog pathway regulators influence cervical cancer cell proliferation, survival and migration. Biochem Biophys Res Commun 425: 64-69, 2012. 
43. Koo CY, Muir KW and Lam EW: FOXM1: From cancer initiation to progression and treatment. Biochim Biophys Acta 1819: 28-37, 2012.

44. Laoukili J, Alvarez M, Meijer LA, Stahl M, Mohammed S, Kleij L, Heck AJ and Medema RH: Activation of FOXM1 during G2 requires cyclin A/Cdk-dependent relief of autorepression by the FOXM1 N-terminal domain. Mol Cell Biol 28: 3076-3087, 2008.

45. Wierstra I: Cyclin D1/Cdk4 increases the transcriptional activity of FOXM1c without phosphorylating FOXM1c. Biochem Biophys Res Commun 431: 753-759, 2013.

46. Xue J, Zhou A, Tan C, Wu Y, Lee HT, Li W, Xie K and Huang S: Forkhead box M1 is essential for nuclear localization of glioma-associated oncogene homolog 1 in glioblastoma multiforme cells by promoting importin-7 expression. J Biol Chem 290: 18662-18670, 2015.

47. Pignot G, Vieillefond A, Vacher S, Zerbib M, Debre B, Lidereau R, Amsellem-Ouazana D and Bieche I: Hedgehog pathway activation in human transitional cell carcinoma of the bladder. Br J Cancer 106: 1177-1186, 2012.

48. Gialmanidis IP, Bravou V, Amanetopoulou SG, Varakis J, Kourea $\mathrm{H}$ and Papadaki $\mathrm{H}$ : Overexpression of hedgehog pathway molecules and FOXM1 in non-small cell lung carcinomas. Lung Cancer 66: 64-74, 2009

49. Bourboulia D and Stetler-Stevenson WG: Matrix metalloproteinases (MMPs) and tissue inhibitors of metalloproteinases (TIMPs): Positive and negative regulators in tumor cell adhesion. Semin Cancer Biol 20: 161-168, 2010.
50. Bourdeanu L and Luu T: J Adv Pract Oncol 5: 246-260, 2014

51. Wainberg ZA and Drakaki A: The importance of optimal drug sequencing in metastatic colorectal cancer: Biological rationales for the observed survival benefit conferred by first-line treatment with EGFR inhibitors. Expert Opin Biol Ther 15: 1205-1220, 2015.

52. Wen N, Wang Y, Wen L, Zhao SH, Ai ZH, Wang Y, Wu B, Lu HX, Yang H, Liu WC and Li Y: Overexpression of FOXM1 predicts poor prognosis and promotes cancer cell proliferation, migration and invasion in epithelial ovarian cancer. J Transl Med 12: 134, 2014.

53. Cui D, Chen X, Yin J, Wang W, Lou M and Gu S: Aberrant activation of Hedgehog/GLI1 pathway on angiogenesis in gliomas. Neurol India 60: 589-596, 2012.

54. Chen JS, Huang XH, Wang Q, Huang JQ, Zhang LJ, Chen XL, Lei J and Cheng ZX: Sonic hedgehog signaling pathway induces cell migration and invasion through focal adhesion kinase/AKT signaling-mediated activation of matrix metalloproteinase (MMP)-2 and MMP-9 in liver cancer. Carcinogenesis 34: 10-19, 2013.

55. Hwang J, Kang MH, Yoo YA, Quan YH, Kim HK, Oh SC and Choi YH: The effects of sonic hedgehog signaling pathway components on non-small-cell lung cancer progression and clinical outcome. World J Surg Oncol 12: 268, 2014.

56. Moeini A, Cornellà $\mathrm{H}$ and Villanueva A: Emerging signaling pathways in hepatocellular carcinoma. Liver Cancer 1: 83-93, 2012. 